Conf- $940135--5$ SAND- $-93-8675 C$

RECFIVED

APR 211994

OSTI

\title{
A SERVO-MECHANICAL LOAD FRAME FOR IN SITU, NON- INVASIVE, IMAGING OF DAMAGE DEVELOPMENT
}

T.M. Breunig, M.C. Nichols, and J.S. Gruver, Center for Materials and Applied Mechanics, Sandia National Laboratories, Livermore, CA 94551-0969.

J.H. Kinney and D.L. Haupt, Lawrence Livermore National Laboratory, Livermore, CA 94550.

\section{ABSTRACT}

The X-ray tomographic microscope (XTM) is a non-invasive X-ray imaging instrument for characterizing a material's microstructure threedimensionally with microscopic spatial resolution. We have designed a servomechanical load frame for use with the XTM which will allow imaging of samples under load. The load frame is capable of generating tensile or compressive forces up to $15.6 \mathrm{kN}$ with a design system stiffness of $8.76 \times 10^{8} \mathrm{~N} / \mathrm{m}$. The test specimen can be rotated through $360^{\circ}$, without induced bending or torque. Torqueless motion is accomplished by synchronously rotating the grips on precision bearings with an accuracy of $0.01^{\circ}$. With this load frame it will be possible, for the first time, to image the initiation and accumulation of internal damage $(0.5 \mu \mathrm{m}$ detectability) formed in a $6 \mathrm{~mm}$ diameter specimen during the application of a monotonic or low frequency cyclic load. This is accomplished by interrupting the test and maintaining a fixed load (or displacement) during the non-invasive XTM data collection procedure. This paper describes the in situ load frame design, experimental capabilities, and preliminary results from a study of crack closure in the aluminum-lithium alloy, 2090.

\section{BACKGROUND}

If ceramic materials, in the form of composites, coatings, or monolithic components, are to be utilized effectively, the intrinsic mechanisms associated with the failure process must be understood. To successfully design ceramic composite structures for high performance, environmentally aggressive applications, knowledge of the general relationships between the composite's macromechanical properties and those of the micromechanical fiber, matrix and fiber/matrix interface properties will be crucial. Estimation of the safe operational lifetime of such structures will also require a fundamental understanding of the damage initiation and evolution process. To date, research directed at obtaining a

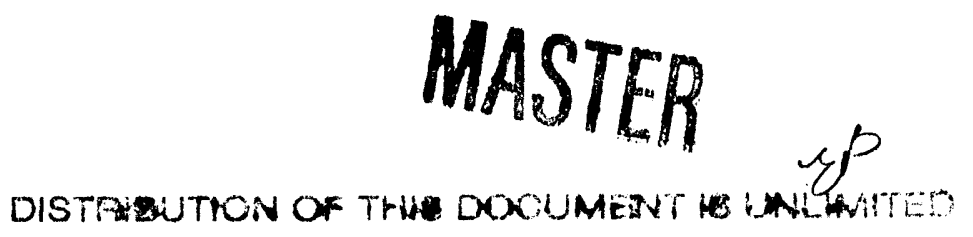


fundamental understanding of failure in ceramic and metal matrix composites has dealt mainly with engineering systems, and has focused on building engineering data bases, creating new fibers with better high temperature properties, and controlling interfacial reactions between fiber and matrix during processing. Although the macromechanical response has been monitored in these studies, relatively little quantitative information exists about the micromechanisms of damage nucleation and growth in these structures. Fundamental studies on mechanics in these systems are difficult because of processing variability and the complexity of commercial fiber architectures make interpretations of mechanical deformation results difficult and often contradictory. Damage in composite materials frequently initiates by fiber fracture, matrix cracking, interface cracking/debonding, delaminations or by a combination of these modes. These forms of damage cannot be readily detected by using surface or nondestructive techniques (like radiography and ultrasound) because of their sensitivity and resolution limitations as well as the structural complexity of such microstructures.

Current methods of evaluating the damage state of a composite material are based upon stiffness loss measurements, acoustic emission and surface replication $[1,2]$. The stiffness of a $\mathrm{CMC}$, however, is not unique to a given damage state and inferences concerning damage accumulation cannot be specific enough to guide physically-based modeling. Damage is frequently anisotropic in nature due to the directionality of the defects present. In many composite structures, insignificant stiffness changes occur until immediately prior to catastrophic failure of a sample [3]. This, combined with sample-to-sample variability in microstructure and mechanical properties, means that internal damage levels and the remaining life of a structure cannot be precisely quantified by stiffness loss techniques. Acoustic emission events monitored during deformation cannot be directly correlated with their source type and location because of microstructural complexity. Because damage often initiates internally in composite materials, it is not possible to use surface techniques as an effective monitoring technique.

Until recently, the oniy way to three-dimensionally analyze the microstructure of an opaque material was time consuming serial sectioning, a destructive technique that does not track the development of damage at specific locations within the same sample, and is acknowledged to be artifact prone [4]. Serial sectioning also requires that the load be removed before examination and any residual stresses will be changed by the preparation of the specimens. X-ray computed tomography is uniquely suited for monitoring damage accumulation since the incremental changes in internal microstructure can be examined within a single sample multiple times during the course of an experiment [5-7]. For most composites, the size scale of the damage features of interest (e.g., fiber, matrix and interphase cracking) is less than $10 \mu \mathrm{m}$, high resolution computed tomography is required to provide the resolution and sensitivity required.

It has long been known that the linear X-ray attenuation coefficient is a sensitive measure of elemental composition and density. Compositional and microstructural changes in a sample from one position to the next appear as differences in the attenuation coefficient. We have developed a technology to make absolute and precise X-ray attenuation measurements on exceedingly small volume elements within a sample. This technology is known as X-ray 
tomographic microscopy, or XTM. The highest spatial resolution $(1-2 \mu \mathrm{m})$ is obtained using monochromatic (i.e., single energy) synchrotron radiation, and is limited to inspection of small (at present less than $6 \mathrm{~mm}$ diameter) specimens. Hundreds of slices can be recorded simultaneously using XTM, and this volume of material can be observed repeatedly throughout the course of a deformation experiment. The three-dimensional data set allows the spatial distribution of features and their associated interactions in a continuous volume of material to be evaluated and thus provides the capability to image the microstructure in a particular sample multiple times before, during, and after mechanical loading or fracture. Because XTM is three-dimensional and non-invasive it has the potential to enhance our understanding of failure in advanced composite materials [8-10]. For the first time it will be possible to experimentally correlate observed microstructural damage to mechanical properties in the same sample. The limited quantities and extremely high costs of many developmental materials make it very important to extract as much information as possible from each specimen examined. XTM's noninvasive nature provides a means to maximize the deformation and microstructural information available from these materials.

\section{RESULTS AND DISCUSSION}

Many material characterization methods developed for monolithic metals and ceramics are not valid for composite materials because of their anisotropy and inhomogeneous microstructure. The macromechanical response of composite materials also exhibit a complex dependence on the microstructure present, the processing method used to develop the microstructure and the way damage is initiated and accumulated. Unlike monolithic materials, damage in composite microstructures frequently initiates internally and the associated changes in local properties may not significantly change a composite's macroscopic properties until a critical number density of structural components have been damaged. To detect and follow the internal damage formed during the application of mechanical loads, an in situ loading (tension and compression) stage has been developed to be used with the XTM. This will allow the three-dimensional detection of damage initiation and accumulation at various stages of a structures deformation history. The ability to nondestructively determine the evolution characteristics of these damage states under in situ conditions will play a critical role in the development of physically-based deformation and damage response models for complex material systems.

A prototype version of the load frame demonstrated that XTM could be performed using a sample under load [6,7]. These previous in situ XTM studies were performed on a laboratory X-ray source and at the Cornell High Energy Synchrotron Source (CHESS). Our studies demonstrated that it was possible to examine the interactions of fatigue crack faces in an Aluminum-Lithium monolithic specimen as a function of the applied load. This early version of a load frame for in situ XTM used a pneumatic actuator to generate forces up to $90.8 \mathrm{~kg}$ and used a polycarbonate stand-off tube to support the applied load. The stand-off tube completely encircled the sample and allowed the specimen to be viewed over a 360 degree range without obstruction from $x$-ray opaque supports. A problem with this design is the significant loss of $\mathrm{X}$-ray photon flux in the polycarbonate 
stand-off tube, $35.6 \%$ at the $\mathrm{Ag} \mathrm{K} \alpha$ peak $(22.1 \mathrm{keV})$. The decrease in photon flux resulted in increased time for data collection and measured linear attenuation coefficients measured were relative rather than absolute. The polycarbonate standoff mateilal also limited the systems stiffness to $1.4 \times 10^{7} \mathrm{~N} / \mathrm{m}$. The maximum sample size that can be examined in this type of system is very limited. This very simple design does not provide a means of controlling or monitoring the sample displacement during a test, but has provided valuable proof-of-concept information.

To overcome the limitations on sample size, relative absorption coefficients, and lack of precise position control, a servo-mechanical load frame was developed. Figure 1 is a schematic drawing of the in situ load frame and it's control system shown with the XTM control and data collection systems. In Figure 1 the $x$-ray path starts at the source, shown at the left of the figure. The $x$ ray photons pass through the sample that is positioned by rotation devices in the load frame. The vertical position of the sample in the X-ray beam can be adjusted to locate the region of interest in the sample's gauge section. The sample is initially translated out of the $\mathrm{x}$-ray path, and an image is obtained of the incident beam, $\mathrm{I}_{0}$. The sample is then moved into the $\mathrm{x}$-ray beam and the photons transmitted through the specimen are collected by the XTM system. This image provides the values of $I$ at a given angular orientation. The sample is rotated by discrete angular increments through 180 degrees and at each position the values of $I$ and $I_{0}$ are collected. These images are then used to reconstruct the threedimensional image of the absolute attenuation coefficients. The in situ load frame controls are also connected to the XTM data collection and control computer. The two systems must communicate to avoid collecting XTM images while the load frame is changing the force or displacement applied to the specimen.

Figures $2 \mathrm{a}$ and $2 \mathrm{~b}$ are photographs of the in situ load frame. To obtain optimum XTM imaging conditions it is imperative that the specimen's position is stable, and repeatable to a higher precision than the detectability limits. This system was designed to be capable of generating tensile and compressive forces up to $15.6 \mathrm{kN}$. A high torque micro-stepping motor is used to drive a precision ball screw linear actuator that loads the test specimen. A micro-stepping motor was selected for this application to provide high precision motion and closed-loop control. In open loop positioning mode, one motor step causes a linear position change of $0.0625 \mu \mathrm{m}$. In closed-loop position control, using the optical encoder on the micro-stepping motor for position feed back, the actuator positioning accuracy is $0.397 \mu \mathrm{m}$. The test specimen must be rotated though 360 degrees without induced bending or torque while under load. To transfer the force generated into the test specimen, grips are attached to the rotation and load transfer mechanisms on the upper and lower support plates. Torqueless motion is accomplished by synchronously rotating the grips on precision bearings with an accuracy of 0.01 degrees. The grip mounts have also been aligned to avoid specimen bending during the rotation procedure. A four post design was used to provide maximum stiffness and uniform support to prevent bending during specimen rotation. The load frame was designed to have a stiffness (spring constant) of $8.76 \times 10^{8} \mathrm{~N} / \mathrm{m}$ with the upper support plate at the top of the four support posts. Specimen displacement measurements can not be made with an 
extensometers that is mounted across the gauge section. This type of extensometer would interfere with the XTM data collection. To make displacement measurements a non contacting extensometer is used. Another method for monitoring displacement in a stiff system is to measure from the upper and lower grips to stable references.

In Figure $2 b$, the system is shown with a set of threaded specimen tensile grips. Most conventional specimen grip types can be used in this system. An exception would be bending fixtures that orient the specimen horizontally. It is possible to adjust the height of the upper support plate by moving the locking collars on the four posts. This adjustment allows a variety of specimen lengths and gripping arrangements to be used. A micro-stepping motor is used to position the specimen height in the $\mathrm{x}$-ray beam. This motor must be capable of lifting the entire load frame except the horizontal positioning mechanism. To avoid using an excessively large motor or counter-weights for this purpose, a set of constant force springs were placed on two sides of the system.

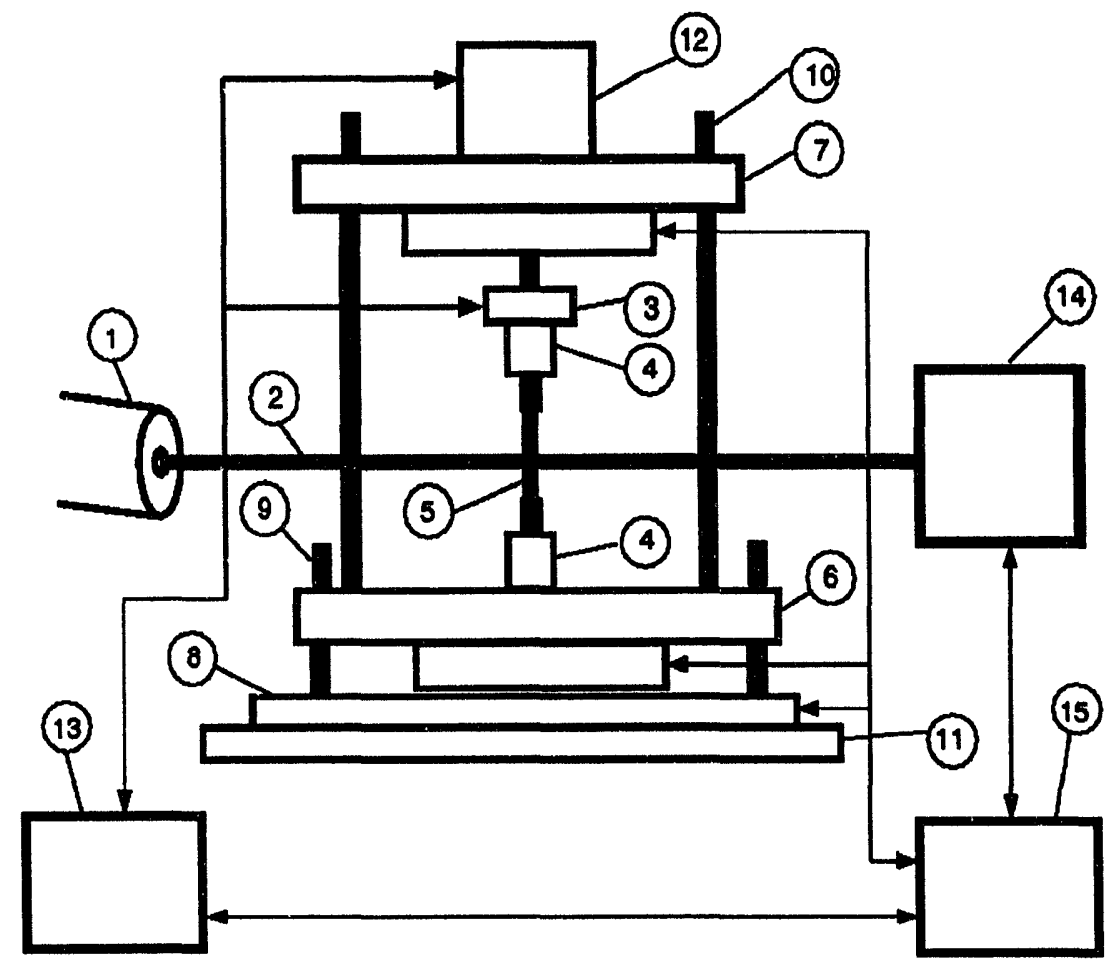

Figure 1. Load Frame System Schematic. Ballooned items are: 1 - X-ray source; 2 - X-ray beam path; 3 - load cell; 4 - specimen grips; 5 - test specimen; 6 - lower support platen; 7 - upper support platen; 8 - horizontal positioning mechanism; 9 vertical positioning mechanism; 10 - four support posts; 11 - stage mounting plate; 12 - linear actuator and drive system; 13 - load frame control/data collection computer; 14 - XTM detector system; 15 - XTM control/data collection computer. 
The data collection sequence is initiated by the load frame's control computer. After sending a control signal, the closed-loop feed back system is used to control the linear actuators motion. This system can be operated in load, strain or stroke control mode. To ensure the XTM image quality, the frame's control mode is changed to maintain specimen displacement. Prior to XTM data collection the load is also monitored and allowed to stabilize. After successful completion of the deformation command, the a signal is sent by the load frame's control computer to the XTM's control computer to allow image collection to begin. If, at any time during the image collection process, either control computer detects a fault condition, the image collection is stopped.

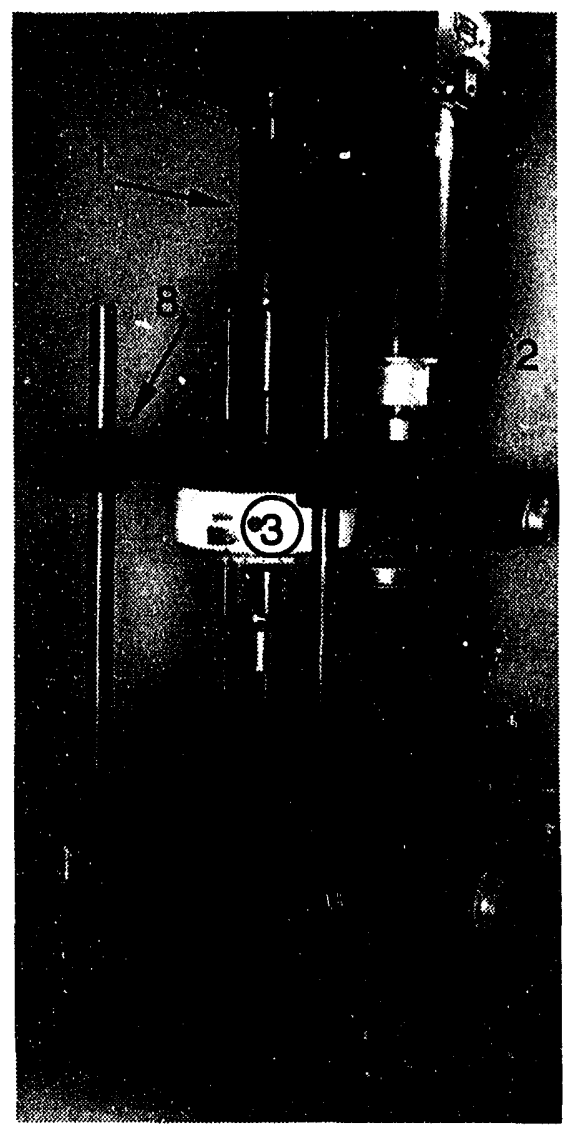

(a)

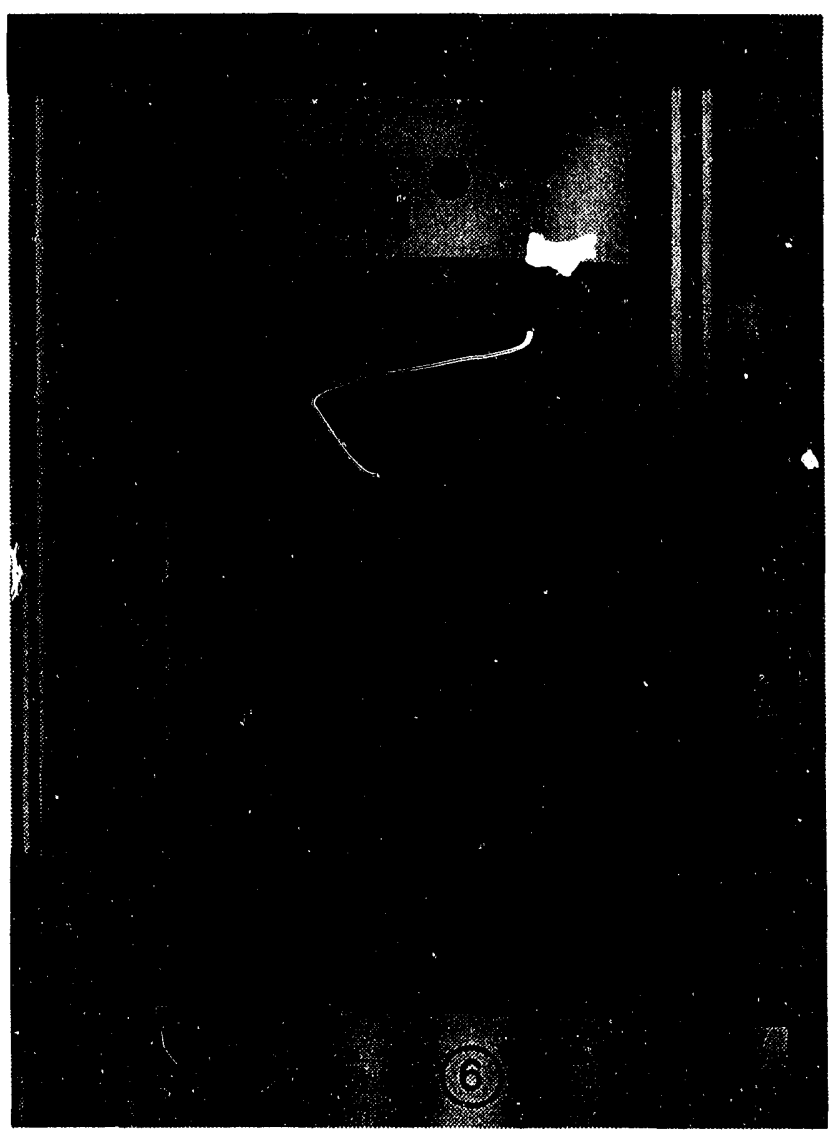

(b)

Figure 2. Photographs of a) the entire in situ load frame system and b) a close-up of the sample test region. Ballooned items are: 1 - ball screw linear actuator; 2 micro-stepping motor; 3 - synchronized rotation stages; 4 - load cell; 5 - sample; 6 - constant force springs; 7 - vertical position micro-step motor; 8 - lock collars. 
A sequence of XTM scans were performed using the prototypic version of the load frame with limited deformation control and monitoring. Figure 3 a shows a typical digital radiograph collected with an applied load of $800 \mathrm{~N}$. The projection direction is parallel to the crack plane and perpendicular to the load axis. Even though a crack is present and is being held open, it is not visible in the radiographs. When a tomographic section parallel to the load axis is examined (Figure 3b), the crack path appears to be very non-planar. This specimen was used to evaluate the effects of crack face interactions as a function of the applied load. This sample was non-invasively imaged at 11 loads, ranging from 0 to $800 \mathrm{~N}$.

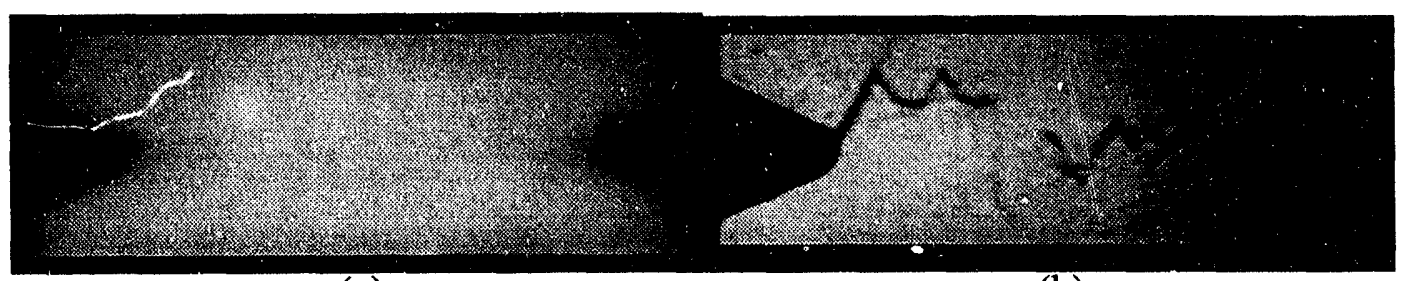

(a)

(b)

Figure 3. a) Typical digital radiograph of a fatigue cracked Al-Li sample at 800 $\mathrm{N}$. b) A Tomographic sections $(6.15 \mu \mathrm{m}$ thick) parallel to the loading direction at $800 \mathrm{~N}$ shows the fracture path non-planarity [11].

\section{SUMMARY}

The capability to perform XTM with an in situ applied load on a composite material has not existed prior to development of this system. By XTM imaging of a composite material with an applied in situ load, the fiber locations and orientations due to deformation are maintained, and cracks and other defects which otherwise might be closed will be held open. The type and size of microstructural features quantifiable from the XTM data sets is dependent on the total volume examined, the attainable spatial resolution and detectability, and the phase contrast. The region examined with XTM is the central zone of the gauge section where extensometry measurements are normally made during mechanical deformation evaluation. The volume of material examined is expected to approach $1,000 \mathrm{~mm}^{3}$ and could contain as many as $8 \times 10^{9}$ volume elements, a statistically significant sampling of the materials microstructure. The ability to use monochromatic radiation allows optimization of contrast between phases in a multi-component material system. Details about the optimum specimen crosssection size and optimum phase contrast of the XTM system can be found elsewhere [9]. Some of the microstructural features that can be measured include: matrix porosity, matrix cracking, fiber orientation, fiber cracking, fiber/matrix interface cracking, spacing and physical location of cracks, volume of features, crack and fiber surface areas, surface orientations and surface spacings. The ability to map the three-dimensional location of these internal damage features and directly correlate a change in mechanical response with the initiation and/or evolution of a critical local number density of damage features is not possible with any other technique. 


\section{ACKNOWLEDGMENTS}

This work is supported by the U.S. Department of Energy under the auspices of DE-AC04-76DP00789 at SNL/CA, and W-7405-ENG-48 at LLNL.

\section{REFERENCES}

1. W.S. Johnson, "Fatigue Testing and Damage Development in Continuous Fiber Reinforced Metal Matrix Composites," NASA TM100628, 1988.

2. B.F. Sorensen, and R. Talreja, "Analysis of Damage in a Ceramic Matrix Composte,", in Int. J. Damage Mechanics, Vol. 2, 1993, pp. 246-271.

3. R.Talreja, "Stiffness Properties of Composite Laminates With Matrix Cracking and Interior Delamination," Engineering Fracture Mechanics, Vol. 25, No. 5/6, 1986, pp.751-762.

4. E.R. Weibel, "Measuring Through the Microscope: Development and Evolution of Stereological Methods," J. Microscopy, Vol. 155, Pt 3, 1989, pp. 393-403.

5. J.H. Kinney, T.M. Breunig, T.L. Starr, D. Haupt, M.C. Nichols, S.R. Stock, M.D. Butts, and R.A. Saroyan, "X-ray Tomographic Study of Chemical Vapor Infiltration Processing of Ceramic Composites," Science, Vol. 260, 7 May 1993, pp. 789-792.

6. T.M. Breunig, S.R. Stock, S.D. Antolovich, J.H. Kinney, W.N. Massey, and M.C. Nichols, "A Framework for Relating Macroscopic Measures and Physical Processes of Crack Closure Illustrated by a Study of AluminumLithium Alloy 2090," Fracture Mechanics: Twenty-Second Symposium (Volume I), ASTM STP 1131, H.A. Ernst, A. Saxena, and D.L. McDowell, Eds., American Society for Testing and Materials, Philadelphia, 1992, pp. 749-761.

7. T.M. Breunig, S.R. Stock and R.C. Brown, "Simple Load Frame for in situ Computed Tomography and X-ray Tomographic Microscopy", J. Materials Evaluation, Volume 51, April, 1993, pp. 596-600.

8. J.H. Kinney, S.R. Stock, M.C. Nichols, U. Bonse, T.M. Breunig, R.A. Saroyan, R. Nusshardt, Q.C. Johnson, F. Busch, and S.D. Antolovich, "Nondestructive Investigation of Damage In Composites Using X-Ray Tomographic Microscopy", J. of Materials Research, Vol. 5, No. 5, (May) 1990, pp. 1123-1129.

9. J.H. Kinney and M.C. Nichols, "X-ray Tomographic Microscopy (XTM) Using Synchrotron Radiation," Annual Reviews of Materials Science, 1992, pp. 121-152.

10. E. Zywicz, J.H. Kinney, M.L. Sattler, T.M. Breunig, and M.C. Nichols, "Heterogeneous Fiber Microstructures and Their Influence on Failure," J. Microscopy, Volume 169, Pt 2, February 1993.

11. J.H. Kinney, M.C. Nichols, U. Bonse, S.R. Stock, T.M. Breunig, A. Guvenilir, and R.A. Saroyan, "Nondestructive Imaging of Materials Microstructures Using X-ray Tomographic Microscopy," in Advanced Tomographic Imaging Methods For The Analysis of Materials, J.L. Ackerman, W.A. Ellingson, eds., Materials Research Society Symposium Proceedings, Vol. 217, 1990, pp. 81-95. 

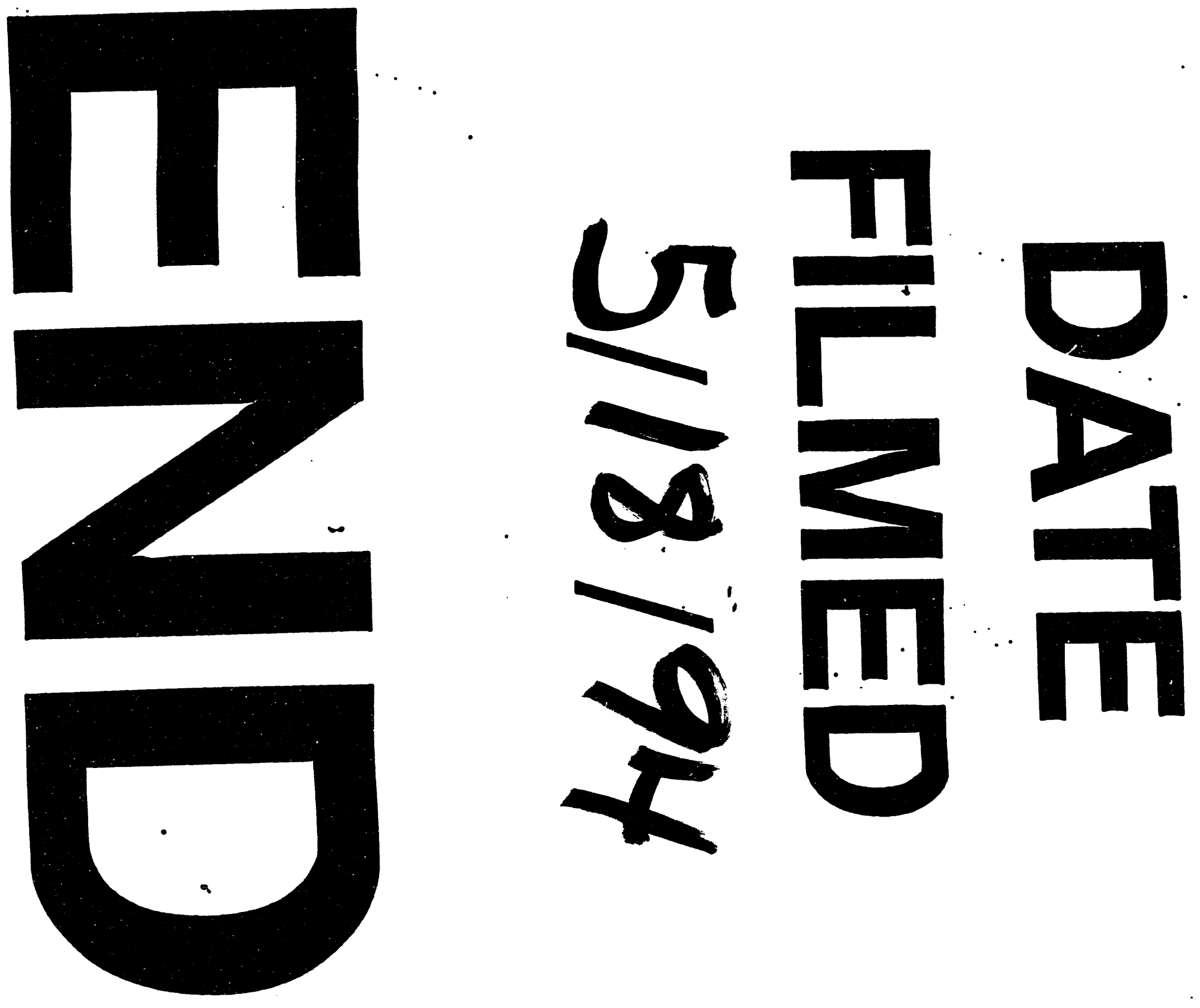
